\title{
rs1344706 polymorphism of zinc finger protein 804a (ZNF804a) gene related to the integrity of white matter fiber bundle in schizophrenics
}

\author{
JIAN ZHOU ${ }^{1}$, QUAN BAO ${ }^{2}$, SHUANG LIANG ${ }^{3}$, HONG GUO $^{4}$, XIN MENG $^{1}$, GUANGFENG ZHANG $^{1}$ and PING LI $^{5}$ \\ ${ }^{1}$ Department of MRI, The Third Affiliated Hospital of Qiqihar Medical University, Qiqihar, Heilongjiang 161000; \\ ${ }^{2}$ Department of MRI, Hongqi Hospital Affiliated to Mudanjiang Medical University, Mudanjiang, \\ Heilongjiang 157011; ${ }^{3}$ Department of Radiology, Hongqi Hospital Affiliated to Mudanjiang Medical University, \\ Mudanjiang, Heilongiiang 157000; ${ }^{4}$ Department of Radiology, The First Hospital of Qiqihar; \\ ${ }^{5}$ Department of Psychiatry, Qiqihar Medical University, Qiqihar, Heilongjiang 161000, P.R. China
}

Received March 16, 2020; Accepted December 7, 2020

DOI: $10.3892 /$ etm.2021.10210

\begin{abstract}
Genetic factors play an important role in the pathogenesis of schizophrenia (SZ), and the zinc finger protein $804 \mathrm{a}$ (ZNF804a) gene has been considered to be a risk gene for schizophrenia. In the present study, the correlation between rs1344706 polymorphism of ZNF804a gene and the integrity of white matter in schizophrenic cases was explored. A total of $60 \mathrm{SZ}$ patients and 100 healthy controls (HC) were included to undergo head MRI. According to the genotyping of rs1344706 in $\mathrm{ZNF804a}$, the subjects in each group were divided into a normal allele and risk allele-carrying group. The imaging data were preprocessed by PANDA software, and thefractional anisotropy (FA) of each subject was calculated. With SPM8 software, age and years of education were considered as covariates, and diagnosis as well as genotype (AA, GG/AG) were considered as intergroup factors. Four groups of FA images were analyzed by two-factor analysis of variance. The FA value of the right posterior radiocrown in the patient group was lower than that in the control group, and the difference was statistically significant. The FA value of the right lower frontal occipital tract and the right upper radiocrown in the $\mathrm{G}$ allele carrier group was lower than that in the A allele homozygous group. There was detection of an interaction between the FA value of the splenium of corpus callosum, the body
\end{abstract}

Correspondence to: Xin Meng, Department of MRI, The Third Affiliated Hospital of Qiqihar Medical University, 27 Taishun Street, Tiefeng, Qiqihar, Heilongjiang 161000, P.R. China

E-mail: mengxinmx0203@126.com

Abbreviations: SZ, schizophrenia; HC, healthy controls; ZNF804a, zinc finger protein 804a; FA, anisotropic fraction; DTI, diffusion tensor imaging; EGFR, epidermal growth factor receptor

Key words: schizophrenia, neuroregulatory protein 1, single nucleotide polymorphism, diffusion tensor imaging part of the corpus callosum and the right cingulate tract. In the present study, it was demonstrated that the rs1344706 GG/AG genotype of the ZNF804a gene locus in SZ patients suffered from abnormal structure in a specific region of the brain. This finding indicated that the rs1344706 single nucleotide polymorphism of the ZNF804a gene may affect the integrity of the white matter of the brain in SZ patients and may be involved in the pathophysiological mechanism of SZ.

\section{Introduction}

Schizophrenia (SZ) belongs to a group of serious mental diseases whose etiology is not completely known. The key clinical characteristics of this disease include perceptual, thinking, emotional and behavioral disorders $(1,2)$. The lifetime prevalence rate is approximately $1 \%$. The onset age of SZ is early and the disease has a chronic progression (3). Progressive aggravation or deterioration, accompanied by social dysfunction along with high disability rate and death rate can create a great burden to the society (4). At present, the pathogenesis of SZ is unclear, however results obtained from prior studies have revealed that genetic factors play an important role in the pathogenesis of SZ $(5,6)$.

With the development of molecular genetics, numerous genes have been revealed to be closely related to the pathogenesis of SZ (7). It has been revealed that the zinc finger protein 804a (ZNF804a) gene polymorphism was closely related to the susceptibility of schizophrenia and the efficacy of antipsychotics $(8,9)$. ZNF804a gene can control the formation of synapses, the proliferation of neurites and the migration of neurons. It plays an important role in the development of central nervous system (10). ZNF804a gene can regulate the expression of schizophrenia-related genes, such as dopamine D2 receptor, catecholamine oxidative methyl transferase, and phosphodiesterase 4B gene (11). ZNF804a which is located on chromosome 8p12-21, acts on the epidermal growth factor receptor (EGFR) family. It spans over 1.4 trillion bases and encodes 15 types of proteins. ZNF804a has been also revealed to play a role in white matter development and SZ 
susceptibility (12). Other studies revealed that the G-A allele mutation may increase the risk of SZ and affect the cognitive function of SZ patients $(13,14)$. It has also been revealed that the genetic defects and neurodevelopmental abnormalities of SZ were related to the dysfunction of glutamatergic neurons (15). The mutation of the ZNF804a gene in some SZ patients leads to the dysregulation of the glutamatergic synapse and nerve fiber, which leads to the abnormal development and function of the nerve fiber, and affects the development of brain white matter (16). In addition, it has been revealed that ZNF804a can regulate GABA energy transmission by pre-synaptic ErbB4 receptor (17). Any changes to the ZNF804a gene sequence may lead to abnormal development of GABAergic interneurons, and the abnormal signal transmission of these neurons may be a step in the pathogenesis of SZ $(13,18)$. These studies indicated that ZNF804a gene abnormality may affect the development of white matter in the brain. This may be related to the pathogenesis of SZ. These previous studies also revealed that any abnormality in white matter plays an important role in the pathogenesis of SZ. At present, diffusion tensor imaging (DTI) is the only non-invasive method used for observing and tracking the white matter fiber bundle (19). The fractional anisotropy (FA) of water molecules is the proportion of anisotropic components of water molecules in the total diffusion tensor, and its variation range is from 0 to 1 (20). DTI reflects the integrity of white matter and the abnormality of white matter fiber bundle by measuring the FA value (21). It has been revealed that the FA value is decreased in patients suffering from disorder in myelination of the central nervous system (22). This observation demonstrated that the FA value is an abnormal sensitive factor in the measurement of myelination. A decrease in the FA value reflects the abnormality of white matter fiber bundle, such as the decrease of axon density and number, the abnormality of oligodendrocyte and myelin lecithin (23). According to these findings, the application of DTI technology can better clarify the changes of brain white matter.

Several researchers have revealed a wide range of brain areas with abnormal white matter in SZ patients. These areas include the corpus callosum, the cingulate tract, the upper and lower longitudinal tracts, the lower fronto-occipital tract and the radial crown (24-26). A previous study demonstrated that compared with $\mathrm{HC}$, the FA value of white matter in SZ patients is substantially decreased. These areas include bilateral posterior radial crowns, bilateral posterior optic nerve internal capsule, bilateral posterior thalamic radiation, left anterior radial crowns, left upper longitudinal bundle, left sagittal plane, right cerebral foot and knee of corpus callosum (27).

Previous studies on ZNF804a polymorphism revealed that the white matter density of the anterior limb of the inner capsule and the connectivity of the DTI structure in the same region of the TT homozygote, the risk gene of rs1344706, was decreased in healthy subjects $(28,29)$. These results revealed that ZNF804a not only affected the migration of neurons, the growth of axons and myelin sheath, but also was related to the decrease of white matter density and integrity in human subjects (30). The decrease in the FA value of the anterior cingulate in SZ patients with rs1344706 allele indicates that ZNF804a mutation may negatively affect the anterior cingulate in SZ patients (31). However, for the ZNF804a site rs1344706, it has been revealed that the GG homozygote of the ZNF804a site rs1344706 was related to the increased risk of early onset of SZ. It has also been suggested that the mutation of rs1344706 in ZNF804a gene may affect the cognitive function of SZ patients (32). There is also research revealing that ZNF804a site rs1344706 has no significant effects on the cognitive ability of SZ patients. It has been reported that genetic variation related to different pathways may affect the brain structure and function of SZ patients (33). However, the exact association between rs1344706 polymorphism of the ZNF804a gene locus and the whole brain white matter integrity of SZ patients is not clearly understood. In addition, the internal mechanism of rs1344706 and the whole brain white matter integrity of SZ patients is not clear. There have been few studies on the interaction between rs1344706 single nucleotide polymorphism of the ZNF804a gene and brain white matter integrity of SZ patients, as well as the physiological roles in cell differentiation, proliferation, and inflammatory processes, which may be part of MS/SZ common pathophysiological processes that participate in the pathophysiological mechanism of SZ (34).

\section{Materials and methods}

Research subjects. In total, 160 subjects, including 76 males and 84 females, aged 14 to 50 years (average age, $42.1 \pm 4.3$ years) were enrolled in the present study, diagnosed and treated at the Department of MRI of the Third Affiliated Hospital of Qiqihar Medical University (Qiqihar, China) from May 2018 to February 2019. There were 60 SZ patients and 100 healthy controls (HC). Inclusion criteria for the SZ group were as follows: The diagnosis of mental illness was confirmed by structured clinical interview using the DSM IV classification system (35). All patients with SZ were assessed with the Brief Psychiatric Rating Scale (BPRS) (36). In the HC group, there was no disease history diagnosed by DSM-IV axial workers and no disease history diagnosed by first-degree relatives. No psychiatric drugs were taken in the previous month. The exclusion criteria were as follows: History of major physical diseases, unstable physical diseases, IQ less than 70; autism or extensive developmental disorders; or claustrophobia. The study was approved by the Ethics Committee of The Third Affiliated Hospital of Qiqihar Medical University. Signed written informed consents were obtained from the patients and/or guardians.

Genotyping. Peripheral venous blood $(2 \mathrm{ml})$ was collected on an empty stomach at 8:00 am on the day of collection. Plasma was removed using low-speed centrifugation $(500 \mathrm{x} \mathrm{g}$ for $10 \mathrm{~min}$ ) at $37^{\circ} \mathrm{C}$. Red blood cell lysate containing $150 \mathrm{~mm} / 1$ $\mathrm{NH} 4 \mathrm{Cl}, 10 \mathrm{mmol} / 1 \mathrm{KHC} 03$ and $0.1 \mathrm{mmol} / 1 \mathrm{EDTA}$ was used to separate white blood cells. After $6 \mathrm{~h}$ of treatment with SDS lysate + proteinase $\mathrm{K}$, proteins were extracted using phenol/chloroform. After centrifugation at 2,000 x g for $5 \mathrm{~min}$ at $37^{\circ} \mathrm{C}$, the supernatant was collected, and the genomic DNA was precipitated with $75 \%$ ethanol. The extracted DNA was stored at $-80^{\circ} \mathrm{C}$ for later use. Venous blood samples $(2 \mathrm{ml})$ were collected, and the single nucleotide polymorphism (SNP) typing kit (product no. HNSV-01; Sigma-Aldrich; Merck KGaA) was used to detect 160 samples for 22 SNP 
Table I. Demographic and clinical data.

\begin{tabular}{|c|c|c|c|c|c|c|}
\hline \multirow{3}{*}{$\frac{\text { Index }}{\text { Sex, M/F }}$} & \multicolumn{2}{|c|}{$S Z(n=60)$} & \multicolumn{2}{|c|}{$\mathrm{HC}(\mathrm{n}=100)$} & & \\
\hline & AA $(n=24)$ & GG/AG $(n=36)$ & $\mathrm{AA}(\mathrm{n}=58)$ & GG/AG $(n=42)$ & \multicolumn{2}{|c|}{ P-value } \\
\hline & $11 / 24$ & $4 / 36$ & $25 / 58$ & $21 / 42$ & $\chi^{2}=4.451$ & 0.220 \\
\hline Age (years) & $24.1 \pm 6.0^{\mathrm{a}}$ & $26.5 \pm 8.2^{\mathrm{a}}$ & $34.7 \pm 8.9^{\mathrm{a}}$ & $31.8 \pm 10.0^{\mathrm{a}}$ & $F=16.566$ & $<0.05$ \\
\hline Education years & $12.0 \pm 4.4^{\mathrm{a}}$ & $13.1 \pm 4.2^{\mathrm{a}}$ & $14.5 \pm 4.6^{\mathrm{a}}$ & $15.4 \pm 5.0^{\mathrm{a}}$ & $\mathrm{F}=3.428$ & 0.019 \\
\hline Course of disease (mouth) & $10.5 \pm 6.3$ & $11.0 \pm 6.0$ & & & $\mathrm{t}=0.361$ & 0.719 \\
\hline BPRS score & $57.8 \pm 23.2^{\mathrm{a}}$ & $51.8 \pm 18.1^{\mathrm{a}}$ & $25.3 \pm 5.8^{\mathrm{a}}$ & $25.9 \pm 6.4^{\mathrm{a}}$ & $\mathrm{F}=59.312$ & $<0.05$ \\
\hline
\end{tabular}

${ }^{a} \mathrm{P}<0.05$. BPRS, Brief Psychiatric Rating Scale; HC, healthy control group; SZ, schizophrenia group.

genotyping. In the present study, only the G/A genotype at rs1344706 of ZNF804a gene was used. The PCR primers were as follows: rs1344706 forward, 5'-AAACCTTTGGAATGG TGCCTTGA-3' and rs1344706 reverse, 3'-CTTATTGCTGGG GGCAGTCTCTAC-5'.

The PCR conditions were as follows: First denaturation at $95^{\circ} \mathrm{C}$ for $2 \mathrm{~min}$, then denaturation at $94^{\circ} \mathrm{C}$ for $20 \mathrm{sec}$, annealing at $65^{\circ} \mathrm{C}$ for $40 \mathrm{sec}$, extension for $1.5 \mathrm{~min}$ at $72^{\circ} \mathrm{C}$ for 11 cycles; then the annealing temperature of each cycle was decreased to $59^{\circ} \mathrm{C}$, and finally denaturation at $94^{\circ} \mathrm{C}$ for $20 \mathrm{sec}$, annealing at $59^{\circ} \mathrm{C}$ for $30 \mathrm{sec}$, and extension for $1.5 \mathrm{~min}$ at $72^{\circ} \mathrm{C}$ for 24 cycles.

Interpretation of results: The alleles determined by the analysis were $\mathrm{G}$ and $\mathrm{A}$ alleles, which were divided into AA, AG and GG genotypes. The genotype and diagnosis were divided into four groups, including the SZ AA group (A allele control group of SZ patients, 24 people), SZ-GG/AG group (G-risk allele carrier group of SZ patients, 36 people), HC-AA group (A allele control group of HC, 58 people) and HC-GG/AG group (G-risk allele carrier group of $\mathrm{HC}, 42$ people).

Brain MRI examination methods. All subjects were scanned with MRI when they were enrolled in the study. Using the 65 layers scanned by the GE Discovery MR750w 3.0T MRI scanner. The thickness of the layer was $2 \mathrm{~mm}^{3}$, the layer spacing was $1 \mathrm{~mm}$, the voxel size was $2 \mathrm{~mm}^{3}$, and the diffusion gradient was applied in 25 directions, $\mathrm{B}=1000 \mathrm{smm}$.

Processing of experimental data and statistical analysis. The standardized FA images of each subject were obtained, and registered on a $1 \times 1 \times 1 \mathrm{~mm}^{3}$ standard spatial image mni-152. We used Pipeline for Analyzing Brain Diffusion Images DTI (PANDA software) (http://www.nitrc.org/projects/panda) in order to preprocess the data. The steps included head movement and eddy current correction, removal of motion artifacts caused by head movement, removing excess scalp and brain tissue, and fitting a tensor model by least squares method to calculate and obtain the FA value. Subsequently, the FA brain map was calculated and smoothed by $6 \mathrm{~mm}^{3}$ half height and full width Gaussian kernel for the next step of analysis and statistics. SPSS 13.0 (SPSS, Inc.) was used for statistical analysis. Chi square test, two-sample unpaired t-test and two-factor factorial design analysis of variance (ANOVA) were used for data statistical analysis. Scheffe's post hoc test was used following ANOVA. Diagnosis (SZ and
HC) and genotype (AA, GG/AG) were used as intergroup factors. Two-factor ANOVA (GRF correction, voxel level $\mathrm{P}<0.05$, regional level $\mathrm{P}<0.05$ ) was carried out on four groups of FA images with SPM8 software (https://www.fil.ion.ucl. ac.uk/spm/software/spm8/), with diagnosis (SZ and HC) and genotype (AA and GG/AG) as intergroup factors, and age and years of education as covariates. $\mathrm{P}<0.05$ was considered to indicate a statistically significant difference.

\section{Results}

Statistical results of the clinical data in the present study. In total, 160 subjects were divided into four groups: SZ-AA group (A allele control group of SZ patients), SZ-GG/AG group ( $\mathrm{G}$ risk allele carrier group of SZ patients), HC-AA group (A allele control group of HC) and HC-GG/AG group ( $\mathrm{G}$ risk allele carrier group of $\mathrm{HC}$ ). Statistical analysis of demographic and clinical data revealed that there was no significant difference between the four groups with regard to sex and the course of disease between the two genotypes of SZ patients $(\mathrm{P}>0.05)$. There were significant differences in age, education years and BPRS scores $(\mathrm{P}<0.05)$. Detailed demographic data and statistical results are presented in Table I. The distribution of the rs1344706 genotype of ZNF804a gene in all samples was in accordance with the Hardy-Weinberg genetic equilibrium law (16) $(\mathrm{P}>0.05)$, and the selected samples were representative of the population. The secondary allele frequency was $26.7 \%$. The genotype frequency of SZ: $\chi^{2}=0.238$ and $\mathrm{P}=0.626$ (Table II).

DTI analysis of multivariate ANOVA. i) Main effect of diagnosis: The FA value of the right posterior radiocrown in the SZ group was lower than that in HC group, with statistical significance (Table III; Fig. 1); and ii) main effect of the gene: The FA value of the right lower frontal occipital tract and right upper radiocrown in the GG/AG group was lower than that in the AA group, with statistical significance (Table III; Figs. 2 and 3).

\section{Discussion}

In the present study, the rs1344706 polymorphism of ZNF804a gene was compared between a patient group (SZ) and a control group (HC). The results indicated that: i) the main effect of disease factors revealed that the FA value of the right posterior radiocrown in the $\mathrm{SZ}$ group was significantly lower than 
Table II. Hardy-Weinberg equilibrium constant.

\begin{tabular}{|c|c|c|c|c|c|c|c|}
\hline \multirow[b]{2}{*}{ Groups } & \multicolumn{2}{|c|}{ Allele frequency $(\%)$} & \multicolumn{3}{|c|}{ Genotype frequency $(\%)$} & \multirow[b]{2}{*}{$\chi^{2}$} & \multirow[b]{2}{*}{ P-value } \\
\hline & $\mathrm{G}$ & A & GG & $\mathrm{AG}$ & $\mathrm{AA}$ & & \\
\hline SZ & 26.7 & 73.3 & 7.1 & 32.1 & 60.7 & 0.238 & 0.626 \\
\hline $\mathrm{HC}$ & 23.2 & 76.8 & & & & & \\
\hline
\end{tabular}

$\mathrm{G}$ and $\mathrm{A}$ are alleles of rs1344706 at the SNP site of the ZNF804a gene. The chi square test revealed a P>0.05 which was consistent with the Hardy-Weinberg equilibrium law, with no statistical significance. HC, healthy control group; SZ, schizophrenia group.

Table III. Results of two-factor ANOVA.

\begin{tabular}{|c|c|c|c|c|c|}
\hline \multirow[b]{2}{*}{ Brain area } & \multirow[b]{2}{*}{ Voxel no. } & \multicolumn{3}{|c|}{$\begin{array}{l}\text { Maximum difference point } \\
\text { MNI coordinate }\end{array}$} & \multirow[b]{2}{*}{ FA value $^{c}$} \\
\hline & & $\mathrm{x}$ & $\mathrm{y}$ & $\mathrm{z}$ & \\
\hline \multicolumn{6}{|l|}{ Main effect of diagnosis } \\
\hline Right rear radial crown & 42 & 52 & -34 & 20 & 18.04 \\
\hline Right lower frontal screening bundle & 26 & 14 & -86 & -8 & 14.17 \\
\hline Right upper radial crown & 29 & 24 & -8 & 26 & 17.67 \\
\hline \multicolumn{6}{|l|}{ Diagnosis $\mathrm{x}$ gene interaction } \\
\hline Splenium of the corpus callosum & 55 & 22 & -48 & 6 & 16.18 \\
\hline Corpus callosum/right cingulate gyrus & 49 & 12 & -10 & 36 & 12.69 \\
\hline
\end{tabular}

${ }^{a}$ Maximum difference point, the point with the largest FA value in the brain area with statistical difference; ${ }^{b} \mathrm{MNI}$ coordinate, the threedimensional positioning space of human brain developed by the Montreal Institute of Neurology (x, y, z indicate three-dimensional coordinate vectors). ${ }^{\mathrm{c}}$ All the brain areas with statistical difference had a GRF correction $\mathrm{P}<0.05$. MNI, Montreal Neurological Institute; FA, fraction anisotropic.

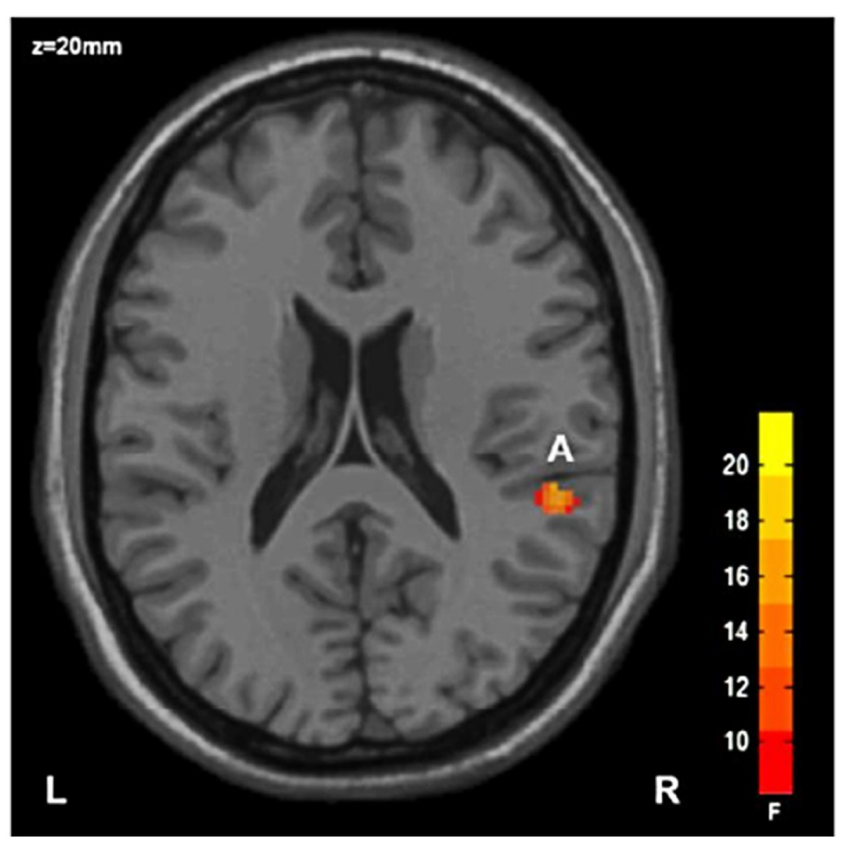

Figure 1. DTI analysis of multivariate ANOVA: Main effect of diagnosis Brain regions with statistically significant difference in the FA value. $L$, represents the left hemisphere; $\mathrm{R}$, represents the right posterior radiocrown of the right hemisphere; A, represents the right posterior radial crown. DTI, diffusion tensor imaging; ANOVA, analysis of variance; FA, anisotropic fraction. that in the HC group; ii) the main effect of rs1344706 polymorphism in the ZNF804a gene revealed that the FA value of the right lower occipital east and right upper radiocrown were significantly lower; and iii) the interaction of the same gene polymorphism in the SZ group revealed that the FA value of the patient group was significantly lower than that in the control group, Compared with the AA control group, the FA value in the splenium of the corpus callosum, the body part of the corpus callosum part and the right cingulate tract in the gene carrier group was significantly decreased. The findings of Cui et al (16) suggest that ZNF804A affects the resting-state functional activation by interacting with COMT and may improve our understanding of the neurobiological effects of ZNF804A and its association with schizophrenia $(16,37)$.

The FA value of the SZ group was significantly lower than that of $\mathrm{HC}$ group. The corona radiata is a radial fiber from the inner capsule to the cerebral cortex (38). It is the main fiber bundle connecting the frontal lobe and other brain areas and regulates sensation and perception. It is mainly related to the cognitive functions of language and memory processing and may be related to positive mental symptoms such as auditory hallucinations (7). Luhrmann et al (38) reported that the FA value of the white matter radiation crown in SZ patients was decreased. Zhao et al also discovered a decrease in the FA value of the radiation crown in SZ patients (39). These findings 


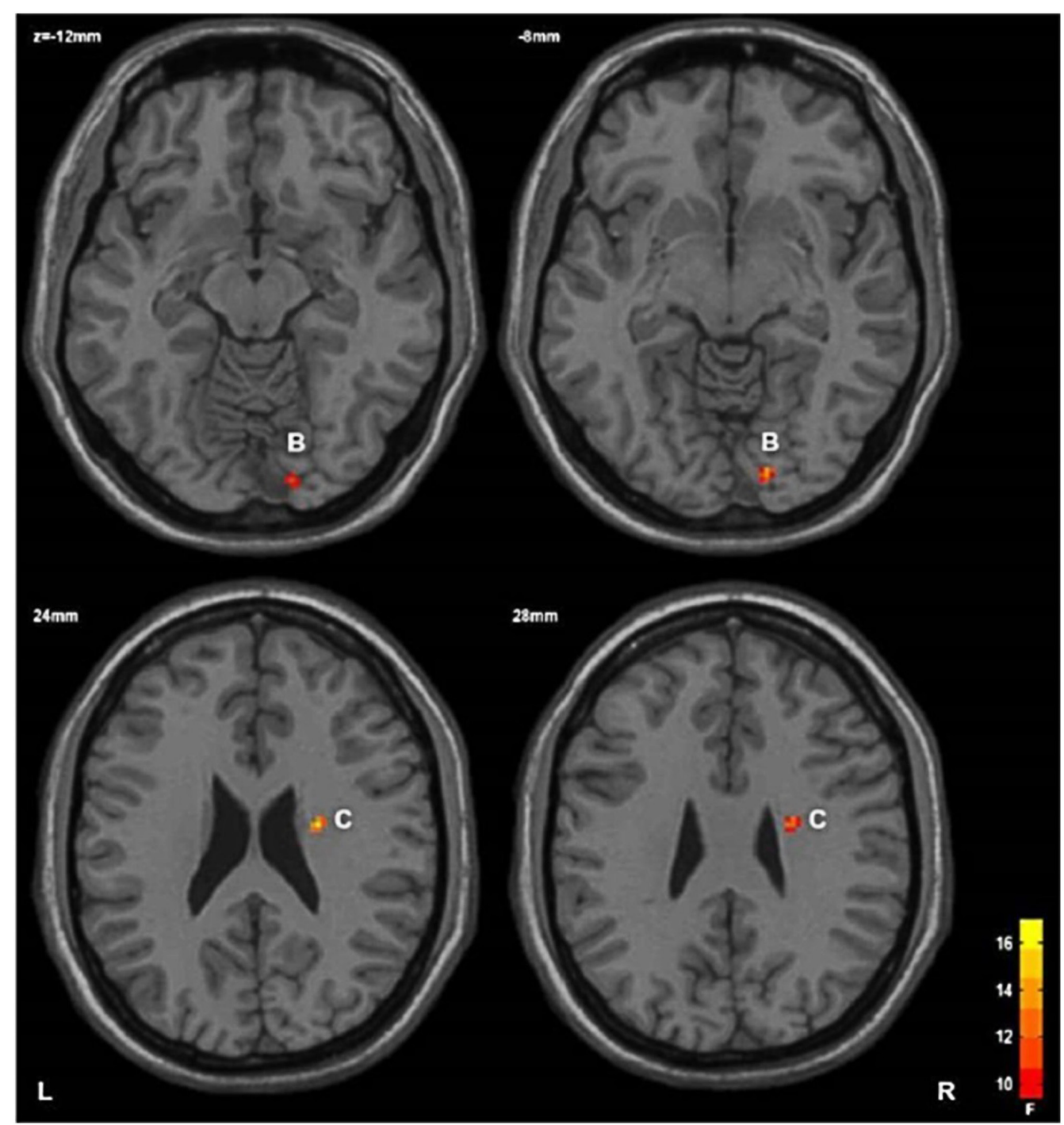

Figure 2. DTI analysis of multivariate ANOVA: Main effect of the gene. Brain regions with statistically significant difference in the FA value. L represents the left hemisphere; R represents the right hemisphere. B represents the right lower frontal occipital tract; C represents the right upper radial crown. DTI, diffusion tensor imaging; ANOVA, analysis of variance; FA, anisotropic fraction.

which were related to the facial expression recognition function of patients, were consistent with our results presented in the present study. These findings suggest that the local white matter fiber bundle and other micro structure abnormalities of the radiation crown may be related to the pathological mechanism of SZ, however there was no gene correlation. Research by Meller et al which focused on the relationship between ZNF804 gene polymorphism and Alzheimer's disease did not evaluate the clinical indications of patients, but our study focused more on the correlation between ZNF804 gene and clinical manifestations of schizophrenia disease, such as MRI examination and analysis of the white matter of the head of patients $(37)$. Other studies $(5,11,12)$ revealed that the FA value of the right anterior radiocrown was positively correlated to the age of onset, although no decrease in the FA value of the SZ group compared with the HC group was reported. These results were inconsistent with the results of the present study. This inconsistence may be related to the age difference and our small sample size. Consistent with a previous study suggesting that functional connectivities within the frontoparietal central executive network are prone to the effect of working memory training (22), the present study provided direct RCT evidence for the effect ofrs1344706 polymorphism of ZNF804a gene related to the integrity of white matter fiber bundle in schizophrenics (39).

In the area where the FA value of the GG/AG genotype in the SZ group demonstrated a significant decrease, we revealed for the first time, to the best of our knowledge, that the FA value of thesplenium of the corpus callosum, the body part of the corpus callosum and right cingulate band in the patient risk gene carrier group was lower than that in the control group. It has been revealed that the dysfunctionality of the glutamatergic system is related to genetic polymorphism (40-42). In the present study, it was theorized that rs1344706 of ZNF804a gene may affect the white matter function in SZ patients through the hypothesis of 'glutamatergic dysfunction'. The corpus callosum is an important nerve fiber bundle connecting the left and right cerebral hemispheres, which is divided into four parts: The splenium part, the body part, the genu part and the rostrum part. It is responsible for the integration of the perception and cognitive functions between the two hemispheres, as well as the transmission of information such as 

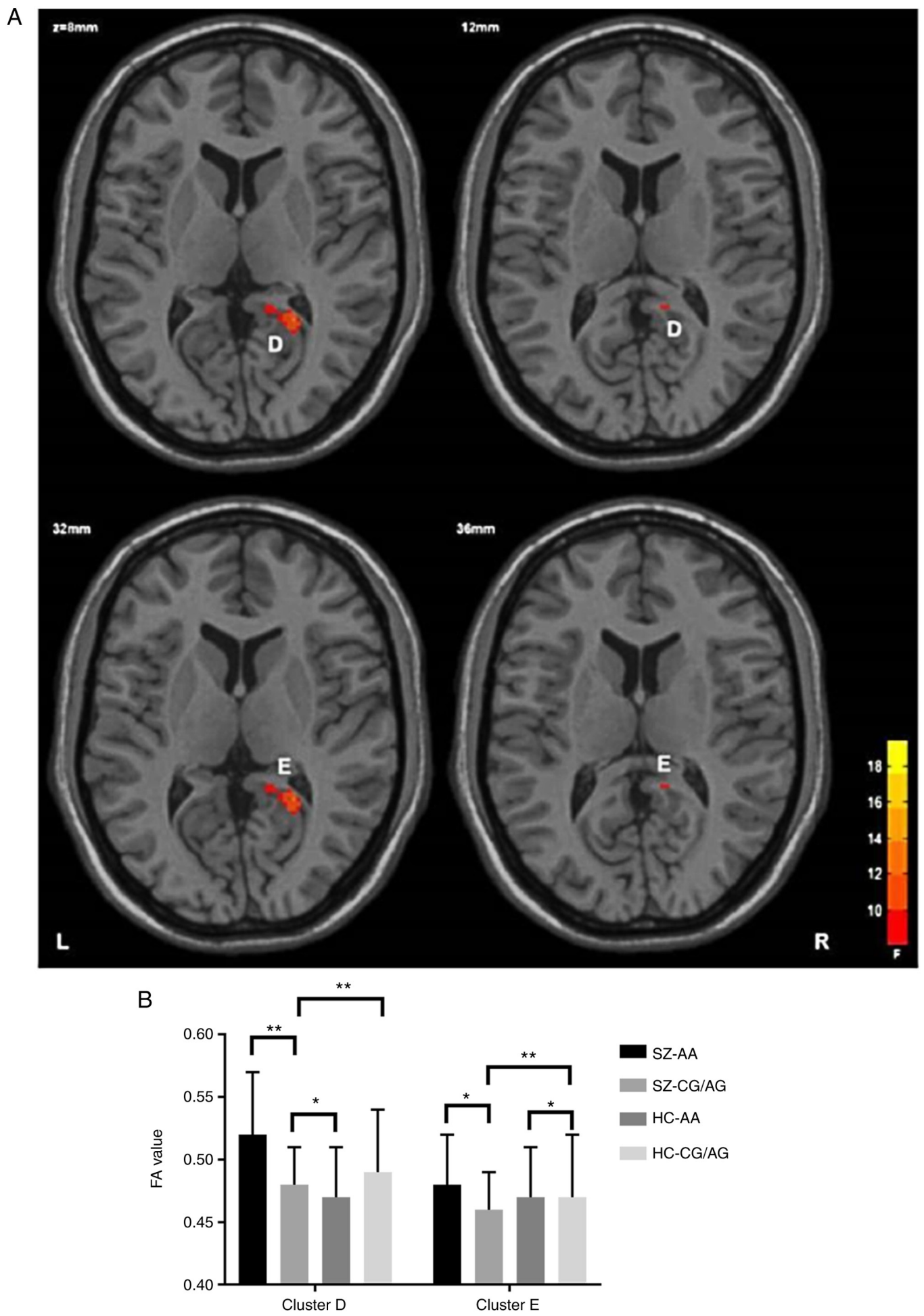

Figure 3. Diagnosis x gene interaction. (A) Diagnosis x gene interaction. Brain regions with a statistically significant difference in the FA value. L represents the left hemisphere; $\mathrm{R}$ represents the right hemisphere; $\mathrm{D}$ represents the splenium of the corpus callosum; E represents the body part of the corpus callosum/right cingulate tract. (B) Diagnosis $x$ gene interaction. The main brain regions with statistically significant differences in the FA value. ${ }^{*} \mathrm{P}<0.05$ and ${ }^{* *} \mathrm{P}<0.01$, as analyzed with Bonferroni correction. FA, anisotropic fraction.

learning, memory and will. It is an important part of human spiritual activities. Among them, the corpus callosum has been revealed to exhibit abnormalities in SZ patients (10). It was revealed in the present study, that the damage of white matter fiber integrity in the corpus callosum and corpus may be related to glutamatergic dysfunction caused by rs1344706 mutation at the ZNF804a site in SZ patients. The cingulate tract is an important part of the limbic green system. It is the white matter fiber connecting the cingulate with the frontal lobe, parietal lobe, parietal region and thalamus. It is important for the functional integrity of SZ patients. The cingulate band is mainly related to emotion regulation, learning ability, attention, memory and other cognitive functions (7). Knöchel et al (27) and revealed that the FA value of white 
matter was decreased in SZ patients. Seitz et al (43) revealed that the FA value of the right cingulate tract was decreased in SZ patients. They speculated that the abnormality of the right cingulate tract may be related to the memory decline and attention deficit in SZ patients, and the white matter change of the right cingulate tract may be caused by the destruction of the myelin sheath. It was suggested that structural abnormalities interact with cognitive function and were related to clinical symptoms. In the present study, it was revealed that the FA value of the right cingulate tract in the risk gene carrier group was lower than that in the control group, indicating that rs1344706 at ZNF804a site may affect the myelination of the central nervous system through the hypothesis of 'glutamatergic dysfunction'. If this process is damaged, it may affect the development of white matter in the brain, thus increasing the possibility of SZ. It was concluded that the rs1344706 single nucleotide polymorphism of ZNF804a gene may affect the integrity of brain white matter in SZ patients.

A previous study revealed that the pathogenesis of SZ is related to the destruction of white matter integrity and the rs1344706 mutation at ZNF804a site (14). This study further indicated that the rs1344706 SNP of ZNF804a gene was related to the integrity of white matter in schizophrenics, and may be related to the pathogenesis of schizophrenia. However, there are still some limitations in the present study. The age and education years of the four groups were not similar. This study used SNP as a covariate, but could not completely exclude (SNP) influence on the results. In the present study, we only addressed the rs1344706 locus and the effect on the structure of white matter. Future studies are required to further explore the interaction between brain function and gene polymorphism. In these future studies, the sample size will be further expanded, more comprehensive analysis of multiple sites that may be related to SZ will be identified, which will aid in further discovering the role of ZNF804a in brain function. In addition, these studies may explore the response of different genotypes to the treatment for schizophrenics, and provide practical basis for precision medicine.

\section{Acknowledgements}

Not applicable.

\section{Funding}

The present study was supported by Clinical Research Foundation of Qiqihar Academy of Medical Sciences (QMSI2019L-22); The General Scientific Research Project of Heilongjiang Education Department (grant no. 2016-KYYWF-0875).

\section{Availability of data and materials}

The datasets used and/or analyzed during the present study are available from the corresponding author on reasonable request.

\section{Authors' contributions}

JZ, QB, SL, HG, XM and GZ conceived and designed the study, and drafted the manuscript. JZ, QB, SL, HG, XM and
PL collected, analyzed and interpreted the experimental data. JZ and XM revised the manuscript for important intellectual content. All authors read and approved the final manuscript.

\section{Ethics approval and consent to participate}

The study was approved by the Ethics Committee of The Third Affiliated Hospital of Qiqihar Medical University. Signed written informed consents were obtained from the patients and/or guardians.

\section{Patient consent for publication}

Not applicable.

\section{Competing interests}

The authors declare that they have no competing interests.

\section{References}

1. Guloksuz S and van Os J: The slow death of the concept of schizophrenia and the painful birth of the psychosis spectrum. Psychol Med 48: 229-244, 2018.

2. Ozaki N: Right treatment for the right schizophrenic patients based on carbonyl stress pathophysiology. Psychiatry Clin Neurosci 72: 2, 2018.

3. Lehman AF, Lieberman JA, Dixon LB, McGlashan TH, Miller AL, Perkins DO and Kreyenbuhl J; American Psychiatric Association; Steering Committee on Practice Guidelines: Practice guideline for the treatment of patients with schizophrenia, second edition. Am J Psychiatry 161 (Suppl 2): 1-56, 2004.

4. Bi XJ, Lv XM, Ai XY, Sun MM, Cui KY, Yang LM, Wang LN Yin AH and Liu LF: Childhood trauma interacted with BDNF Val66Met influence schizophrenic symptoms. Medicine (Baltimore) 97: e0160, 2018.

5. Thyme SB, Pieper LM, Li EH, Pandey S, Wang Y, Morris NS, Sha C, Choi JW, Herrera KJ, Soucy ER, et al: Phenotypic landscape of schizophrenia-associated genes defines candidates and their shared functions. Cell 177: 478-491.e20, 2019.

6. Oh SB, Kim MS, Park S, Son H, Kim SY, Kim MS, Jo DG, Tak E and Lee JY: Clusterin contributes to early stage of Alzheimer's disease pathogenesis. Brain Pathol 29: 217-231, 2019.

7. Bocci V, Zanardia I, Valacchi G, Borrelli E and Travagli V: Validity of oxygen-ozone therapy as integrated medication form in chronic inflammatory diseases. Cardiovasc Hematol Disord Drug Targets 15: 127-138, 2015.

8. Voineskos AN,Lerch JP,Felsky D, Tiwari A, Rajji TK, Miranda D, Lobaugh NJ, Pollock BG, Mulsant BH and Kennedy JL: The ZNF804A gene: Characterization of a novel neural risk mechanism for the major psychoses. Neuropsychopharmacology 36: 1871-1878, 2011.

9. Zhang F, Chen Q, Ye T, Lipska BK, Straub RE, Vakkalanka R, Rujescu D, St Clair D, Hyde TM, Bigelow L, et al: Evidence of sex-modulated association of ZNF804A with schizophrenia. Biol Psychiatry 69: 914-917, 2011

10. Zhou Y, Dong F, Lanz TA, Reinhart V, Li M, Liu L, Zou J, Xi HS and Mao Y: Interactome analysis reveals ZNF804A, a schizophrenia risk gene, as a novel component of protein translational machinery critical for embryonic neurodevelopment. Mol Psychiatry 23: 952-962, 2018.

11. Lencz T, Szeszko PR, DeRosse P, Burdick KE, Bromet EJ, Bilder RM and Malhotra AK: A schizophrenia risk gene, ZNF804A, influences neuroanatomical and neurocognitive phenotypes. Neuropsychopharmacology 35: 2284-2291, 2010

12. Steinberg S, Mors O, Børglum AD, Gustafsson O, Werge T, Mortensen PB, Andreassen OA, Sigurdsson E, Thorgeirsson TE, Böttcher Y, et al: Expanding the range of ZNF804A variants conferring risk of psychosis. Mol Psychiatry 16: 59-66, 2011.

13. Girgenti MJ, LoTurco JJ and Maher BJ: ZNF804a regulates expression of the schizophrenia-associated genes PRSS16, COMT, PDE4B, and DRD2. PLoS One 7: e32404, 2012. 
14. Wu S, Wang P, Tao R, Yang P, Yu X, Li Y, Shao Q, Nie F, Ha J, Zhang R, et al: Schizophrenia-associated microRNA-148b-3p regulates COMT and PRSS16 expression by targeting the ZNF804A gene in human neuroblastoma cells. Mol Med Rep 22: $1429-1439,2020$.

15. Wang Z, Zhang T, Liu J, Wang H, Lu T, Jia M, Zhang D, Wang L and Li J: Family-based association study of ZNF804A polymorphisms and autism in a Han Chinese population. BMC Psychiatry 19: 159, 2019.

16. Cui L, Wang F, Chang M, Yin Z, Fan G, Song Y, Wei Y, Xu Y, Zhang Y, Tang Y, et al: Spontaneous regional brain activity in healthy individuals is nonlinearly modulated by the interaction of ZNF804A rs1344706 and COMT rs4680 polymorphisms. Neurosci Bull 35: 735-742, 2019.

17. Gambarotta G, Garzotto D, Destro E, Mautino B, Giampietro C, Cutrupi S, Dati C, Cattaneo E, Fasolo A and Perroteau I: ErbB4 expression in neural progenitor cells (ST14A) is necessary to mediate neuregulin-1betal-induced migration. J Biol Chem 279: 48808-48816, 2004

18. Zhang R, Lu SM, Qiu C, Liu XG, Gao CG, Guo TW Valenzuela RK, Deng HW and Ma J: Population-based and family-based association studies of ZNF804A locus and schizophrenia. Mol Psychiatry 16: 360-361, 2011.

19. Roberts RM, Mathias JL and Rose SE: Relationship between diffusion tensor imaging (DTI) findings and cognition following pediatric TBI: A meta-analytic review. Dev Neuropsychol 41: 176-200, 2016

20. Commowick O, Fillard P, Clatz O and Warfield SK: Detection of DTI white matter abnormalities in multiple sclerosis patients. Med Image Comput Comput Assist Interv 11: 975-982, 2008

21. Mazal AT, Ashikyan O, Cheng J, Le LQ and Chhabra A: Diffusion-weighted imaging and diffusion tensor imaging as adjuncts to conventional MRI for the diagnosis and management of peripheral nerve sheath tumors: Current perspectives and future directions. Eur Radiol 29: 4123-4132, 2019.

22. Grazzini I, Redi F, Sammartano K and Cuneo GL: Diffusion tensor imaging in idiopathic normal pressure hydrocephalus: Clinical and CSF flowmetry correlations. Neuroradiol J 33: $66-74,2020$.

23. Visser MM, Yassi N, Campbell BC, Desmond PM, Davis SM, Spratt N, Parsons M and Bivard A: White matter degeneration after ischemic stroke: A longitudinal diffusion tensor imaging study. J Neuroimaging 29: 111-118, 2019.

24. Du J, Ma G, Li S, Carl M, Szeverenyi NM, VandenBerg S, Corey-Bloom J and Bydder GM: Ultrashort echo time (UTE) magnetic resonance imaging of the short $\mathrm{T} 2$ components in white matter of the brain using a clinical 3T scanner. Neuroimage 87: 32-41, 2014.

25. Minkner K, Lovblad KO, Yilmaz H, Alimenti A, Sekoranja L, Delavelle J, Sztajzel R and Rüfenacht DA: White matter lesions in watershed territories studied with MRI and parenchymography: A comparative study. Neuroradiology 47: 425-430, 2005.

26. Wu MK, Lu YT, Huang CW, Lin PH, Chen NC, Lui CC, Chang WN, Lee CC, Chang YT, Chen SF and Chang CC: Clinical significance of cerebrovascular biomarkers and white matter tract integrity in Alzheimer disease: Clinical correlations With Neurobehavioral Data in Cross-Sectional and After 18 Months Follow-ups. Medicine (Baltimore) 94: e1192, 2015.

27. Knöchel C, Schmied C, Linden DE, Stäblein M, Prvulovic D, de A de Carvalho L, Harrison O, Barros Junior PO, Carvalho AF, Reif A, et al: White matter abnormalities in the fornix are linked to cognitive performance in SZ but not in BD disorder: An exploratory analysis with DTI deterministic tractography. J Affect Disord 201: 64-78, 2016.

28. Mallas E, Carletti F, Chaddock CA, Shergill S, Woolley J, Picchioni MM, McDonald C, Toulopoulou T, Kravariti E, Kalidindi S, et al: The impact of CACNA1C gene, and its epistasis with ZNF804A, on white matter microstructure in health, schizophrenia and bipolar disorder. Genes Brain Behav 16: 479-488, 2017.
29. Zhang Z, Chen X, Yu P, Zhang Q, Sun X, Gu H, Zhang H, Zhai J, Chen M, Du B, et al: Effect of rs1344706 in the ZNF804A gene on the connectivity between the hippocampal formation and posterior cingulate cortex. Schizophr Res 170: 48-54, 2016.

30. Gurung R and Prata DP: What is the impact of genome-wide supported risk variants for schizophrenia and bipolar disorder on brain structure and function?A systematic review. Psychol Med 45: 2461-2480, 2015.

31. Sprooten E, McIntosh AM, Lawrie SM, Hall J, Sussmann JE, Dahmen N, Konrad A, Bastin ME and Winterer G: An investigation of a genomewide supported psychosis variant in ZNF804A and white matter integrity in the human brain. Magn Reson Imaging 30: 1373-1380, 2012

32. Calabrò M, Mandelli L, Crisafulli C, Nicola MD, Colombo R, Janiri L, Lee SJ, Jun TY, Wang SM, Masand PS, et al: ZNF804A gene variants have a cross-diagnostic influence on psychosis and treatment improvement in mood disorders. Clin Psychopharmacol Neurosci 18: 231-240, 2020

33. Chen X, Zhang Z, Zhang Q, Zhao W, Zhai J, Chen M, Du B, Deng X, Ji F, Wang C, et al: Effect of rs1344706 in the ZNF804A gene on the brain network. Neuroimage Clin 17: 1000-1005, 2017.

34. de Castro-Catala M, Mora-Solano A, Kwapil TR, CristóbalNarváez P, Sheinbaum T, Racioppi A, Barrantes-Vidal N and Rosa A: The genome-wide associated candidate gene ZNF804A and psychosis-proneness: Evidence of sex-modulated association. PLoS One 12: e0185072, 2017.

35. Uher R, Payne JL, Pavlova B and Perlis RH: Major depressive disorder in DSM-5: Implications for clinical practice and research of changes from DSM-IV. Depress Anxiety 31: 459-471, 2014.

36. Schooler NR: Precursors to the PANSS: The BPRS and its progenitors. Innov Clin Neurosci 14: 10-11, 2017.

37. Meller T, Schmitt S, Stein F, Brosch K, Mosebach J, Yüksel D, Zaremba D, Grotegerd D, Dohm K, Meinert S, et al: Associations of schizophrenia risk genes ZNF804A and CACNA1C with schizotypy and modulation of attention in healthy subjects. Schizophr Res 208: 67-75, 2019.

38. Luhrmann TM, Alderson-Day B, Bell V, Bless JJ, Corlett P, Hugdahl K, Jones N, Larøi F, Moseley P, Padmavati R, et al: Beyond trauma: A multiple pathways approach to auditory hallucinations in clinical and nonclinical populations. Schizophr Bull 45 (Suppl 1): S24-S31, 2019.

39. Zhao W, Chen X, Zhang Q, Du B, Deng X, Ji F, Xiang YT, Wang C, Dong Q, Chen C and Li J: Effect of ZNF804A gene polymorphism (rs1344706) on the plasticity of the functional coupling between the right dorsolateral prefrontal cortex and the contralateral hippocampal formation. Neuroimage Clin 27: 102279,2020

40. Zarate CA, Quiroz J, Payne J and Manji HK: Modulators of the glutamatergic system: Implications for the development of improved therapeutics in mood disorders. Psychopharmacol Bull 36: 35-83, 2002.

41. Sanacora G, Zarate CA, Krystal JH and Manji HK: Targeting the glutamatergic system to develop novel, improved therapeutics for mood disorders. Nat Rev Drug Discov 7: 426-437, 2008.

42. Spanagel R, Pendyala G, Abarca C, Zghoul T, Sanchis-Segura C, Magnone MC, Lascorz J, Depner M, Holzberg D, Soyka M, et al: The clock gene Per2 influences the glutamatergic system and modulates alcohol consumption. Nat Med 11: 35-42, 2005.

43. Seitz J, Lyall AE, Kanayama G, Makris N, Hudson JI, Kubicki M, Pope HG Jr and Kaufman MJ: White matter abnormalities in long-term anabolic-androgenic steroid users: A pilot study. Psychiatry Res Neuroimaging 260: 1-5, 2017.

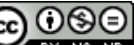

This work is licensed under a Creative Commons Attribution-NonCommercial-NoDerivatives 4.0 International (CC BY-NC-ND 4.0) License. 